\title{
The effect of multivalent cations and Tau on paclitaxel- stabilized microtubule assembly, disassembly, and structure
}

\author{
Cyrus R. Safinya ${ }^{\mathrm{a}, *}$, Peter J. Chung ${ }^{\mathrm{a}}$, Chaeyeon Song ${ }^{\mathrm{a}}$, Youli Li ${ }^{\mathrm{a}}$, \\ Kai K. Ewert ${ }^{\mathrm{a}}$, and Myung Chul Choi ${ }^{\mathrm{b}}$
}

a Materials Department, Physics Department, Molecular, Cellular, and Developmental Biology Department, University of California, Santa Barbara, California 93106, USA

${ }^{\mathrm{b}}$ Department of Bio and Brain Engineering, Korea Advanced Institute of Science and Technology (KAIST), Daejeon 305-701, Republic of Korea

* Corresponding author. Tel +1 8059677298.

E-mail address: safinya@mrl.ucsb.edu

\begin{abstract}
In this review we describe recent studies directed at understanding the formation of novel nanoscale assemblies in biological materials systems. In particular, we focus on the effects of multivalent cations, and separately, of microtubule-associated protein (MAP) Tau, on microtubule (MT) ordering (bundling), MT disassembly, and MT structure. Counter-ion directed bundling of paclitaxel-stabilized MTs is a model electrostatic system, which parallels efforts to understand MT bundling by intrinsically disordered proteins (typically biological polyampholytes) expressed in neurons. We describe studies, which reveal an unexpected transition from tightly spaced MT bundles to loose bundles consisting of strings of MTs as the valence of the cationic counter-ion decreases from $Z=$ 3 to $Z=2$. This transition is not predicted by any current theories of polyelectrolytes. Notably, studies of a larger series of divalent counter-ions reveal strong ion specific effects. Divalent counter-ions may either bundle or depolymerize paclitaxel-stabilized MTs. The ion concentration required for depolymerization decreases with increasing atomic number. In a more biologically related system we review synchrotron small angle x-ray scattering (SAXS) studies on the effect of the Tau on the structure of paclitaxelstabilized MTs. The electrostatic binding of MAP Tau isoforms leads to an increase in the average radius of microtubules with increasing Tau coverage (i.e. a re-distribution of protofilament numbers in MTs). Finally, inspired by MTs as model nanotubes, we briefly describe other more robust lipid-based cylindrical nanostructures, which may have technological applications, for example, in drug encapsulation and delivery.
\end{abstract}

Keywords: Microtubule, multivalent counter-ions, microtubule-associated protein Tau, intrinsically disordered proteins, correlated ion density fluctuations, ion specific effects, SAXS, TEM 


\section{Introduction}

An area of global current interest in the field of soft matter, in particular, as relates to biological systems is the fundamental understanding of interactions and mechanisms underlying lipid and protein based assembly, which lead to hierarchical structures with distinct shapes and functional properties. This interest is driven in part by the current proteomics era where the scientific community is beginning the daunting task of understanding the structures and functions of a large number of self-assembling proteins. In this review we describe experiments designed to understand assembling behavior of paclitaxel-stabilized microtubules (MTs) both in model electrostatic systems and also in the presence of relevant cellular proteins.

Microtubules (MTs) are nanometer scale hollow cylinders (Fig. 1a). They are components of the cytoskeleton of eukaryotic cells [1-4]. The building blocks of MTs are $\alpha / \beta$ tubulin heterodimers, which align head-to-tail to form linear protofilaments (PFs) (a dimer and PF are outlined on the MT cartoon in Fig. 1a). Stabilizing interactions between PFs leads to the curved protein wall. MTs and their dynamical assemblies are utilized by cells for a variety of activities, which include providing tracks for the transport of cargo (e.g. organelles or vesicles containing enzymes, neurotransmitter precursors [5], etc), and formation of the dynamical spindle during the cell cycle [1].

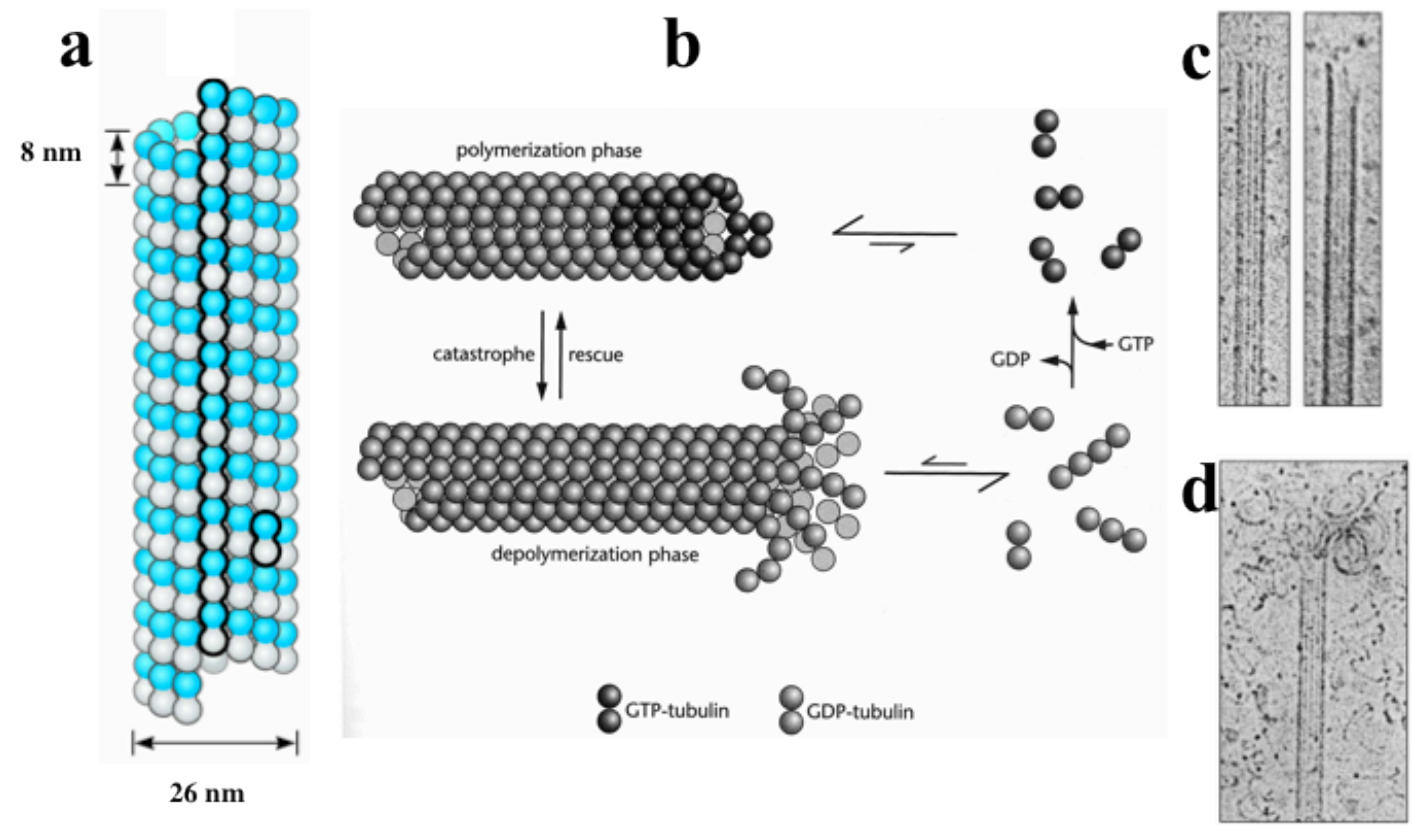

Fig. 1. Structure and dynamics of microtubules. a) Cartoon of a microtubule (MT). A tubulin dimer and protofilament are highlighted. b) Cartoon of MT dynamic instability showing cycling of a microtubule between the growth stage (top, polymerization phase) and the shrinkage stage (bottom, depolymerization phase). In the growth stage the MT contains a GTP-cap (dark spheres) at the growing tip. The GTP-cap is lost during the shrinkage stage where curled PFs rapidly disassemble. c) and d) Cryogenic transmission electron microscopy depicting MT polymerization (c, with straight conformation of PFs within the MT wall) and depolymerization (d, with PFs showing an inside-out curling of PFs). (a) Redrawn from [4]; (b) reprinted by permission from [4]. (c) and (d) reprinted with permission from [6]. Copyright 1991, Rockefeller University Press. 
Microtubules are inherently dynamical and in the absence of stabilizing proteins exhibit dynamic instability where they stochastically switch between periods of growth (polymerization) and periods of shrinkage (depolymerization) (Fig. 1b) [2-4]. Underlying this behavior is the property of the $\alpha / \beta$-tubulin dimer, a two state enzyme (a GTPase) where the $\beta$-tubulin subunit contains a nucleotide site, which is occupied either by GTP or, upon hydrolysis, GDP. PF sections containing GTP-tubulin exhibit a straight conformation whereas PFs with GDP-tubulin are curved. The growth stage of MTs requires a GTP-tubulin cap at the lengthening end to maintain the straight conformation of all PFs within the MT wall (even after hydrolysis of sections downstream of the GTPcap, Fig. 1b top cartoon). The shrinkage stage corresponds to a loss of the GTP-cap (e.g. in regions of the cell with a low local concentration of GTP) resulting in catastrophic rapid disassembly by the inside-out curling of the GDP-PFs (Fig. 1b, lower cartoon). The growth and shrinkage stages have been visualized by cryogenic transmission electron microscopy (TEM) (Fig. $1 \mathrm{c}$ and d) [6].

Microtubules may be stabilized by the addition of the drug molecule paclitaxel commonly used in cancer chemotherapy applications [7-9]. The hydrophobic drug binds to a pocket on the $\beta$-subunit accessible from the side facing the inner MT lumen [10]. Protofilaments containing paclitaxel are kinetically trapped in the straight conformation even after GTP hydrolysis [11,12].

\section{The role of multivalent counter-ions in modulating the interactions between paclitaxel-stabilized microtubules}

Counter-ions suppress electrostatic repulsions between similarly charged rods and in some cases, in particular, for large counter-ion density fluctuations, may produce attractions [13-17]. In considering the interactions between similarly charged rods like DNA, filamentous actin, or microtubules, in the presence of counter-ions, one normally starts with the process of Manning condensation [14]. Counter-ions with valence $Z$ condense on the rod surface when the Manning parameter $\mathrm{M}=\mathrm{Z} \mathrm{l}_{\mathrm{B}} / \mathrm{l}_{\mathrm{o}}>1$. Here, $\mathrm{l}_{\mathrm{o}}$ is the distance between unit charges along the rod and the Bjerrum length $1_{\mathrm{B}}$ (the distance where the Coulomb repulsion between unit charges equals $\mathrm{k}_{\mathrm{B}} \mathrm{T}$ ) is equal to $\mathrm{e}^{2} / \varepsilon \mathrm{k}_{\mathrm{B}} \mathrm{T}=7.1 \AA$ at $\mathrm{T}$ $=300 \mathrm{~K}$ in water with $\varepsilon=80$ (Fig. $2 \mathrm{a}$ ). For example, for DNA $1_{\mathrm{o}}=1.7 \AA$ and $\mathrm{M} \approx 4.17 \mathrm{Z}$. Thus, within Manning theory (i.e. non-linear Poisson-Boltzmann) counter-ions condense on the backbone of DNA until the new renormalized length $\left(1_{0}^{*}\right)$ between unit charges (accounting for the condensed counter-ions) is equal to $1_{\mathrm{B}}$ beyond which counter-ions do not condense as the entropic loss upon condensation is larger than the lowering of the coulomb repulsion energy (Fig. 2a, right cartoon) [14,15]. Counter-ion condensation suppresses the repulsive electrostatic interactions between the rods with increasing counter-ion valence $Z$ by reducing the effective charge per unit length to a fraction $1 /(\mathrm{M})$ of its bare charge per unit length.

It is important to point out that Manning condensation follows from the analysis of the distribution of counterions around an oppositely charged rod using the non-linear Poisson-Boltzmann equation of electrostatics for the two regimes $M>1$ and $M<1$. (The Poisson-Boltzmann equation combines Poisson's equation, relating the gradient squared of the electrostatic potential to the counterion density, together with Boltzmann's distribution equation for the counterions, which at thermal equilibrium relates the counterion density to the exponential of the electrostatic potential energy of the counterion in units of $\mathrm{k}_{\mathrm{B}} \mathrm{T}$ [18]. Thus, the Poisson-Boltzmann equation is inherently nonlinear in the electrostatic potential.) 
Manning condensation theory is inherently a nonspecific phenomenon involving point-like counterions, which does not take into account ion-specific effects arising from the finite size of counterions (i.e. ionic radii) and the thickness of hydration layers around the counterions [14]. Lacking any information on the size of the counterions (which typically dictate the strength of the electrostatic interactions between the ions and water molecules and the thickness of the hydration layer), the theory is not able to predict whether counterions shed hydration layers when coming in contact with anions on the surface of the rod. Within Manning condensation point-like counterions are assumed to be free to diffuse on the rod surface (i.e. they are physisorbed) rather than bond to any anionic region on the rod surface. This is unlike the case where metal ions shed hydration layers and bind specifically within enzymatic pockets.

In the case where the cloud of counter-ions is uniform the interaction between the charged rods remains repulsive (i.e. counter-ion condensation does not neutralize the rod). Counter-ion induced attraction only occurs when the counter-ion cloud has a nonuniform distribution. Fumio Oosawa described the earliest model of attraction between similarly charged rods [13]. In this high temperature model, attraction between rods arises from correlated counter-ion - counter-ion density fluctuations (i.e. similar to the van der Waals attraction where transient dipoles are correlated) (Fig. 2b). More recent low temperature models and simulations show that at sufficiently low temperatures the bound counter-ions form a 1D Wigner crystal [16,17]. In these alternative models attractions arise from counter-ions on adjacent rods developing positional correlations (i.e. staggered rods as shown in Fig. 2c), resulting in a short-range exponentially decaying attractive force, with strength increasing as $\mathrm{Z}^{2}$ and range $\mathrm{Zl}_{\mathrm{o}}$ (= lattice spacing for the 1D Wigner crystal).
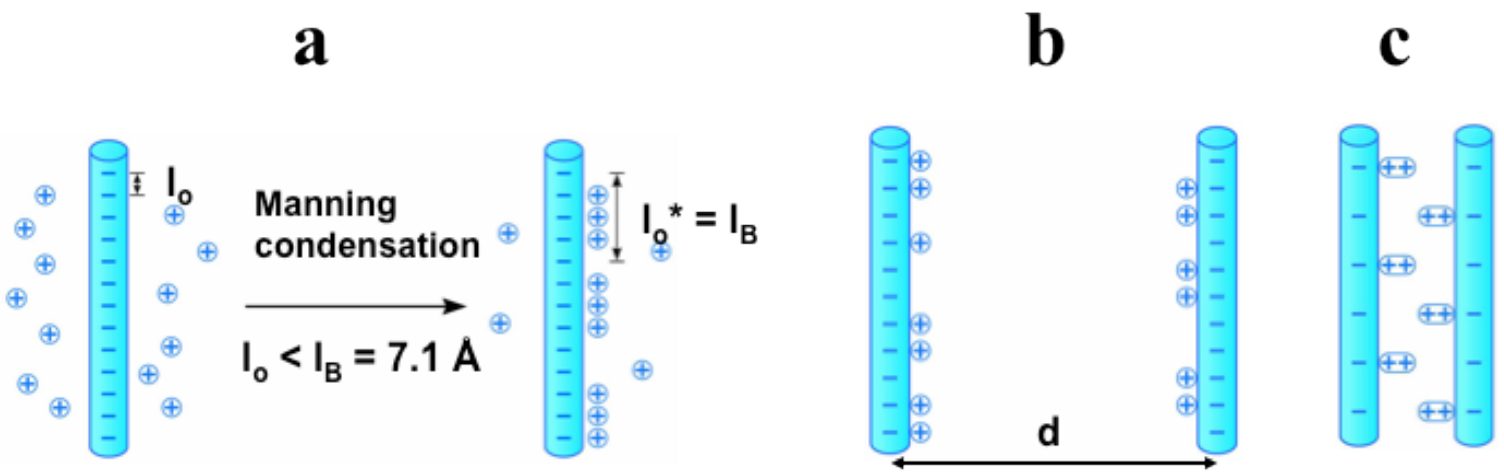

Fig. 2. Cartoon of univalent counter-ion condensation on oppositely charged rods when the bare length $\left(l_{o}\right)$ is less than the Bjerrum length. In mixtures of univalent and multivalent counterions, the latter replace the former for a gain in entropy (i.e. one multivalent ion condensing on the backbone would release $\mathrm{Z}$ univalent ions back in solution with about the same reduction in the electrostatic energy). b) and c) high and low temperature models of counter-ion induced attractions between charged rigid polyelectrolytes with similar charge. In the high temperature model (b) counter-ion fluctuations on one rod (i.e. producing local dipoles) correlate with counter-ion fluctuations in a neighboring rod leading to a dipole-dipole attraction. In the low temperature model (c) the counter-ions form a 1D Wigner crystal on each rod and the correlation between staggered rods (to optimally align oppositely charged regions) leads to a short-range attraction.

For microtubules, the effective distance between unit charges $1_{0}=0.19 \AA$ is artificially small because of the large MT diameter $\approx 26 \mathrm{~nm}$, measured quantitatively by synchrotron small-angle-x-ray-scattering (SAXS) [19-23] (about 10 times larger than the 
diameter of DNA), and a relatively high MT surface charge density $\sigma_{\mathrm{MT}}=-0.66 \mathrm{e} / \mathrm{nm}^{2}$ [10]. A more realistic approach in analyzing the behavior of counter-ions near the smallcurvature MT surface is by approximating it to the behavior of counter-ions near planar surfaces (e.g. models which treat counter-ions near charged membranes). Experiments have shown that attractions between sheets arise for multivalent counter-ions $(Z \geq 2)$ near high charge density $(\sigma)$ surfaces [24-26]. Furthermore, for highly charged surfaces, Monte-Carlo simulations also show clear evidence of attraction for large enough Z [2729].

In this limit of high $\sigma$ and $\mathrm{Z}$, the Gouy-Chapman length $\mathrm{l}_{\mathrm{G}-\mathrm{C}}=1 /\left[2 \pi \mathrm{Z} \mathrm{l}_{\mathrm{B}}(\sigma / \mathrm{e})\right]$ (defined as the height above a charged surface where the electrostatic potential energy of a counter-ion is equal to $\mathrm{k}_{\mathrm{B}} \mathrm{T}$ ), is very small of order angstroms. Physically, $1_{\mathrm{G}-\mathrm{C}}$ corresponds to the approximate thickness of a layer, which contains most of the counterions. Thus, in this limit where $1_{\mathrm{G}-\mathrm{C}}<1_{\mathrm{B}}$, counter-ions form a two-dimensional (2D) ion layer. This is the strong coupling limit clarified by Roland Netz [30]. For 2D systems where $1_{\mathrm{G}-\mathrm{C}}<1_{\mathrm{B}}$ thermal fluctuations are strongly enhanced and Poisson-Boltzmann theory (which works for systems where counter-ions form a uniform 3D cloud) breaks down [31]. High temperature models of membranes with 2D counter-ion layers predict powerlaw long-range attractions (which vanish as $\mathrm{T}$ goes to zero) between high $\sigma$ membranes resulting from correlated in-plane ion density fluctuations [32-35].

In these theoretical models the aforementioned non-linear Possion-Boltzmann equation (which is analytically solvable only in one dimension) was modified in two important ways. First, the equations were linearized (i.e. yielding the Debye-Hückel limit) making it far more tractable [18]. The Debye-Hückel limit is expected to be a valid approximation for small electrostatic potential energies less than $\mathrm{k}_{\mathrm{B}} \mathrm{T}$ (i.e. electrostatic potential of order $25 \mathrm{mV}$ at $\mathrm{T}=300 \mathrm{~K}$ ). Second, in order to obtain short-range attractions the authors modified the standard Debye-Hückel treatment by including in-plane fluctuations of the counterions about a mean counterion density (in the absence of fluctuations, the Debye-Hückel treatment only predicts repulsive forces between membranes).

Another class of low temperature models consider the limit where the $2 \mathrm{D}$ ion layer crystallizes [36-39]. As pointed out by Netz [30] this is analogous to a 2D onecomponent-plasma (i.e. ions near a charge neutralizing uniform background), which is known to crystallize when the plasma parameter (equal to the Coulomb energy between pairs of ions divided by thermal kinetic energy $\left.\mathrm{k}_{\mathrm{B}} \mathrm{T}\right) \approx 125$ [40]. Once again, similar to the case of rods described above, positional correlations between two surfaces with crystalline counter-ion order will produce a short range attraction where the range of the interaction is set by the unit cell lattice constant of the $2 \mathrm{D}$ crystal. In these models attractions are strongest at $\mathrm{T}=0$ and vanish with increasing $\mathrm{T}$.

For MTs, $1_{\mathrm{G}-\mathrm{C}}=3.4 / \mathrm{Z} \AA$ at $\mathrm{T}=300 \mathrm{~K}$ and $\sigma_{\mathrm{MT}}=-0.66 \mathrm{e} / \mathrm{nm}^{2}$ so even for $\mathrm{Z}=2$ one expects the counter-ions to form a 2D ion layer in the liquid state near the MT surface. Thus, one expects large long-wavelength fluctuations in the counter-ion density (about the mean ion distribution) near the MT surface. Correlations between ion fluctuations in neighboring MTs is expected to lead to attractions between MTs. At low temperature, Monte-Carlo simulations in the strong coupling limit confirms the presence of attractions between like charged flat surfaces [30] and between cylinders in the presence of neutralizing multivalent counter-ion layers even in the liquid state [41]. 
a

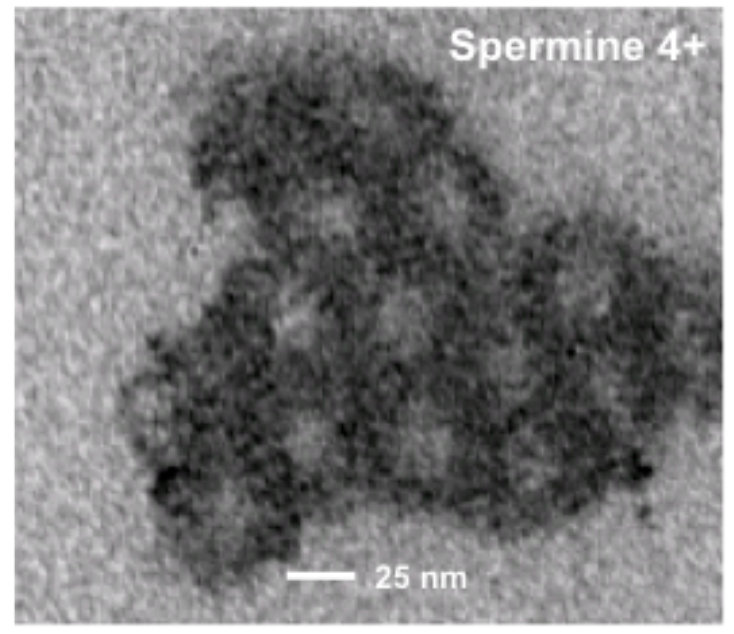

b

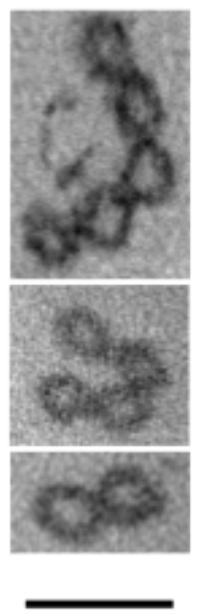

$50 \mathrm{~nm}$

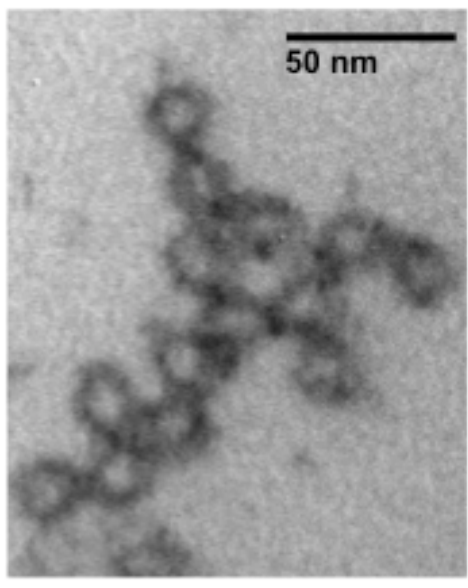

Fig. 3. Plastic embedded TEM cross-sections of microtubule bundles. a) Tightly spaced hexagonal microtubule bundles in the presence of $10 \mathrm{mM}$ spermine $4^{+} . b$ ) and c) Open structured string-like bundles of microtubules in the presence of $100 \mathrm{mM} \mathrm{BaCl}_{2}$. The bundles may be linear (b) or branched (c). Reprinted with permission from [42]. Copyright 2004, National Academy of Sciences, U.S.A..

SAXS and TEM has revealed that counter-ions mediate attractions between paclitaxel-stabilized microtubules [42]. Fig. 3a shows plastic embedded TEM micrographs of cross-sections of MTs bundled with spermine (4+) above a bundling concentration $\approx 1.5 \mathrm{mM}$. The cross-section reveals a tightly spaced $3 \mathrm{D}$ hexagonal bundle. Hexagonal bundles are also observed with other cations with $Z \geq 3$ including trivalent spermidine $(3+)$, and pentavalent (lysine $)_{5}(5+)$, above their bundling concentrations of $7.5 \mathrm{mM}$ and $0.75 \mathrm{mM}$ respectively [42]. In contrast divalent cations $\mathrm{Ca}^{2+}, \mathrm{Ba}^{2+}$ and $\mathrm{Sr}^{2+}$ were found to induce assembly into a string of MTs for concentrations larger than 40 $\mathrm{mM}, 60 \mathrm{mM}$, and $60 \mathrm{mM}$, respectively (Fig. 3b). These bundles also tend to show branched structures (Fig. 3c) [42]. As we discuss in section 3. In addition to bundling mediated by divalents cations there are also unexpected ion-specific effects on the structure of MTs.

Thus, the experiments show that the charge (and likely size and shape) of the multivalent counter-ions modulates MT-MT interactions and lead to bundles with distinct symmetry (hexagonal versus linear). Although there are currently no electrostatic theories, which predict this transition, one may expect it to occur as a consequence of competition between ion-induced short-range attraction and longer ranged repulsion between MTs. The C-terminal tails (CTTs) of the $\alpha$ - and $\beta$-tubulin subunits are known to be highly negatively charged short polyelectrolytes with 18 and 21 residues respectively. This is expected to result in a brush like repulsion with brush height $\mathrm{L}_{\mathrm{CTT}} \approx 3.7 \mathrm{~nm}[43]$. Strong attractions (i.e. due to correlated ion fluctuations described above) for large valence counter-ions $(Z \geq 3)$ appears to overcome the CTT brush repulsion leading to tight hexagonal bundles, whereas, for $Z=2$, comparable attraction and repulsion seems to favor an open bundle structure observed in experiments.

\section{Ion-specific effects in microtubule ordering and depolymerization in the presence of divalent cations}


In the previous section we described how ion-mediated attractions by divalent cations $\mathrm{Ba}^{2+}$ and $\mathrm{Sr}^{2+}$ and $\mathrm{Ca}^{2+}$ induces, above a critical concentration, string-like bundling of MTs where the cross-sectional architecture may be linear or branched (Fig. 3). In this section we describe the finding that a significant number of divalent cations, notably, the biological ions $\mathrm{Mg}^{2+}, \mathrm{Mn}^{2+}$, and $\mathrm{Zn}^{2+}$, and $\mathrm{Co}^{2+}$ (normally absent in vivo), do not bundle paclitaxel-stabilized MTs. The lack of MT bundling is substantiated in SAXS data, with scattering profiles consistent with the form factor of MTs modeled as nanotubes with no evidence of a bundling correlation peak [44]. Instead, above a critical ion concentration, the form factor is replaced by an enhanced and featureless SAXS scattering indicative of depolymerization of paclitaxel-stabilized MTs into curled protofilament rings (Fig. 4a and Fig. 1d where TEM shows MT depolymerization involves inside-our curling of protofilaments into rings).

A summary of the behavior of MT depolymerizing divalent cations is depicted in Fig. 4b. The y-axis is a plot of the MT radius (from fits to the SAXS data [44]) and the x-axis shows the range over which MTs are stable for each cation. For each ion plotted, the end of the color coded arrow corresponds to the highest ion concentration where SAXS data indicates stable MTs. Furthermore, the same color line following the arrow head covers the range over which depolymerization occurs. For example, $\mathrm{Zn}^{2+}$ depolymerizes MTs between $1 \mathrm{mM}$ and $10 \mathrm{mM}, \mathrm{Co}^{2+}$ and $\mathrm{Mn}^{2+}$ between $20 \mathrm{mM}$ and $50 \mathrm{mM}$, and $\mathrm{Mg}^{2+}$ between $50 \mathrm{mM}$ and $100 \mathrm{mM}$. The overall "ion-specific" trend in the data is that divalent cation $\mathrm{Zn}^{2+}$ with the largest atomic number of 30 is the most efficient (i.e. requiring the smallest concentration for MT depolymerization), followed by $\mathrm{Co}^{2+}$ and $\mathrm{Mn}^{2+}$ (with atomic numbers 27 and 25) and finally $\mathrm{Mg}^{2+}$ with the much smaller atomic number of 12 .

The effect of the ions on MT ordering versus depolymerization appears to be dependent on the ionic radius. The bundling inducing cations $\mathrm{Ca}^{2+}, \mathrm{Sr}^{2+}$, and $\mathrm{Ba}^{2+}$ have relatively large radii equal to $0.100 \mathrm{~nm}, 0.118 \mathrm{~nm}$, and $0.135 \mathrm{~nm}_{3}$ respectively. On the other hand, the destabilizing cations, $\mathrm{Zn}^{2+}, \mathrm{Co}^{2+}, \mathrm{Mn}^{2+}$, and $\mathrm{Mg}^{2+}$, have smaller radii ranging from $0.067 \mathrm{~nm}$ to $0.0745 \mathrm{~nm}$ [45]. Smaller radius ions with larger surrounding electric fields are expected to contain larger hydration layers due to the stronger electrostatic association with neighboring water molecules [46,47]. Thus, the larger ions, which tend to shed their hydration layer more easily, may be expected to mediate direct ion contact bonding two neighboring anionic domains on the MT surface leading to bundling. The destabilization of MTs is most likely due to disruption of anionic-cationic amino-acid bonds on neighboring protofilaments [48,49]. The data suggest that the small radii cations with large hydration layers perform the disruption by displacing the cationic amino acid residues involved in the aforementioned ionic bonds stabilizing protofilaments. Noatably, while all four cations have a relatively small ionic radius their efficiency is not directly related to their ionic radius. The most and least efficient ions at MT destabilization $\mathrm{Zn}^{2+}$ (between $1 \mathrm{mM}$ and $10 \mathrm{mM}$ ) and $\mathrm{Mg}^{2+}$ (between $50 \mathrm{mM}$ and $100 \mathrm{mM}$ ) have comparable ionic radii $\approx 0.074 \mathrm{~nm}$ and $0.072 \mathrm{~nm}$, respectively [45]. In contrast, the efficiency of destabilizing cations $\left(\mathrm{Zn}^{2+}, \mathrm{Co}^{2+}, \mathrm{Mn}^{2+}\right.$, and $\left.\mathrm{Mg}^{2+}\right)$ increases systematically with increasing atomic number.

It is interesting to note that under different preparation conditions zinc ions may induce a novel sheet structure consisting of polymerized tubulin in the form of $2 \mathrm{D}$ sheets [50-53]. Aside from forming novel nanostructures these sheets have turned out to be crucial in electron crystallography studies to determine the structure of $\alpha / \beta$ tubulin fixed with paclitaxel. In the experiments described in this review $\mathrm{ZnCl}$ was added to microtubules stabilized with paclitaxel. On the other hand Zinc-sheets are formed by incubating tubulin in the presence of GTP and zinc ions. The GTP state insures that the protofilaments are maintained in the straight conformation. Quite interestingly over longer incubation times the sheets tend to curl up into novel macrotubules. 

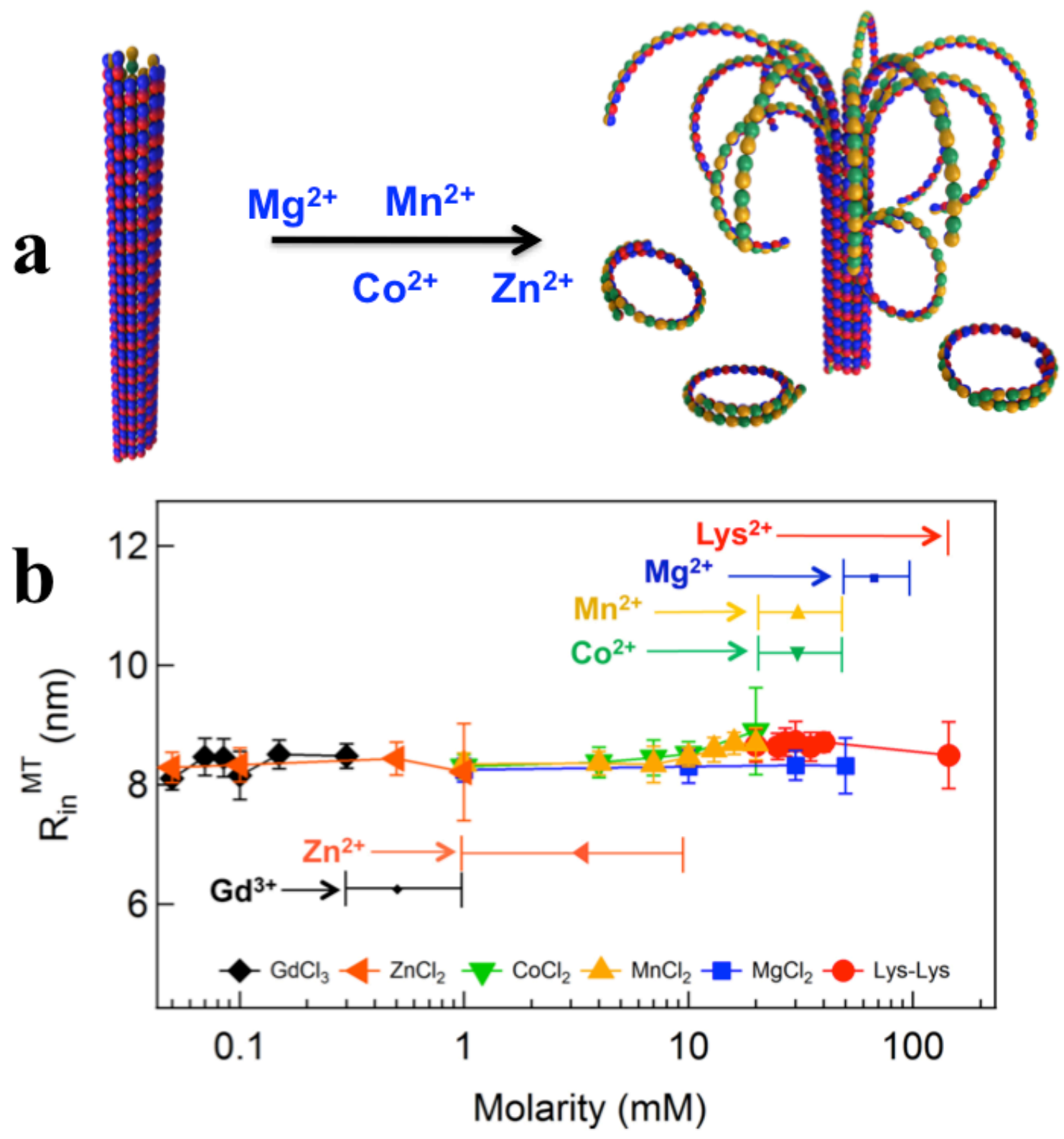

Fig. 4. Ion-specific effects in destabilization of paclitaxel-stabilized microtubules (MTs). a) Cartoon depicting depolymerization of paclitaxel-stabilized MTs. b) Plot of the range of ion concentrations for various divalent cations above which microtubule destabilization sets in. The ends of the color-coded arrows correspond to the highest ion concentration where SAXS data indicate stable MTs. The colored lines following the arrow-heads correspond to the region where MTs depolymerize. The observed trend is that the ion concentration at which MT depolymerization sets in decreases with increasing atomic number although more data are needed to distinguish between $\mathrm{Co}^{2+}$ and $\mathrm{Mn}^{2+}$. The y-axis shows that the MT inner radius (derived from fits to SAXS data) is unchanged in the presence of the different ions. Reproduced from [44] with permission from the Royal Society of Chemistry. 


\section{Microtubule-associated protein Tau controls the diameter of paclitaxel-stabilized microtubules}

As discussed in the introduction microtubules may exhibit dynamic instability in cells involving cycles of growth and rapid shrinkage. Dynamic instability is critical in many cell functions. For example, it enables MTs to search and capture metaphase chromosomes during mitosis before cell division. In mature neurons, which do not divide [5], different isoforms of the microtubule-associated protein (MAP) Tau (Fig. 5) modulate MT dynamic instability by suppressing the rate of catastrophes (i.e. where MTs switch from the growth to the shrinkage stage, see Fig. 1b) while simultaneously enhancing the rate of tubulin assembly during the growth stage [54].

Protein Tau is studied extensively by laboratories worldwide. This interest is driven by the observation that changes in the interaction between protein Tau and MTs (e.g. due to over-phosphorylation of Tau), which lower the fraction of bound Tau leading to increased soluble Tau, enhance MT depolymerization and Tau tangles. Tangles are a hallmark of neurodegenerative diseases including fronto-temporal dementia with Parkinsonism linked to chromosome 17 (FTDP-17), late stage Alzheimer's disease, Pick's disease, supra-nuclear palsy, and chronic traumatic encephalopathy in athletes [55$60]$.
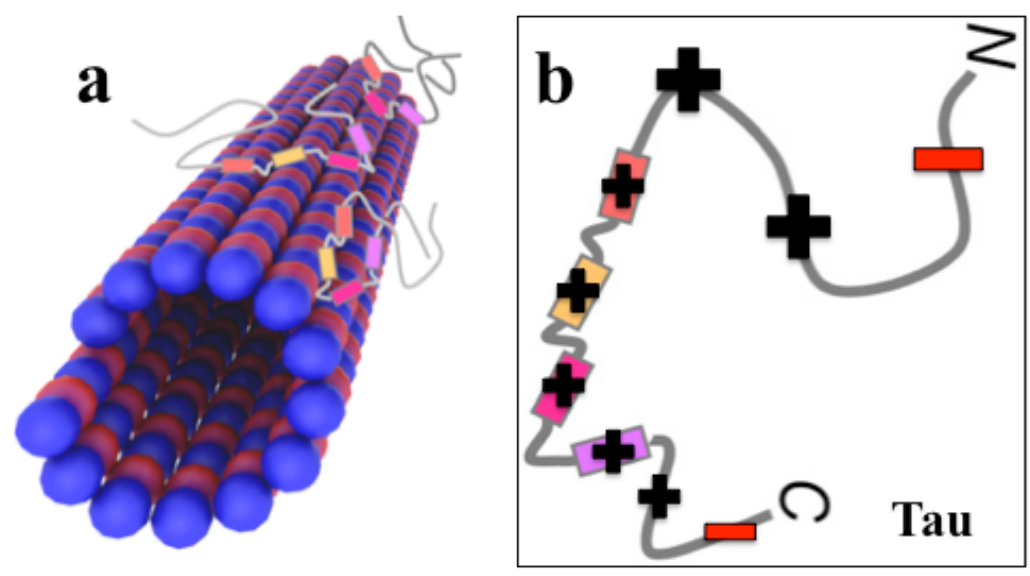

Fig. 5. a) Cartoon of a microtubule (MT) with adsorbed MAP Tau macromolecules. MTs are overall negatively charged while Tau isofroms are overall cationic. b) Cartoon of one of the six human isoforms of Tau showing the charge distribution along the chain. Tau is an intrinsically disordered protein (in this case a polyampholyte containing both cationic and anionic residues). Tau isoforms contain either 3 repeat (3R) or 4 repeat (4R) cationic binding domains (colored rectangular boxes). The isoforms also differ in their $\mathrm{N}$-terminal projection domains, which contain either zero, one, or two 29 amino acid inserts, thereby generating short (S-), medium (M), or long (L-) isoforms.

The six isoforms of human Tau are overall cationic thus binding to and stabilizing microtubules [61]. The MT binding domains of Tau isoforms consist of either 3 or 4 imperfect repeats of 18 amino acids (labeled 3R- or 4R-), and inter-repeat sections of 1314 amino acids, resulting from exclusion or inclusion of exon 10 [62-65]. The N-terminal 
tail consists of an anionic/cationic dipolar projection domain (PD) with little affinity to the MT surface [66,67] followed by a cationic proline rich region (Fig. 5b). The exclusion or inclusion of exon 2 and 3 in the PD leads to the short (S-), medium (M-) and long (L-) isoforms.

In a recent study Synchrotron SAXS was used to quantitatively investigate the assembly structure of paclitaxel-stabilized MTs in the presence of the six Tau isoforms (Fig. 6) [19]. The SAXS data (Fig. 6a) were analyzed by modeling MTs as hollow tubes with inner MT radius $\left(<\mathrm{R}_{\text {in }}{ }^{\mathrm{MT}}>\right)$ [20-23] and wall thickness equal to $49 \AA$ consistent with previously published work [10]. Upon binding to the MT surface Tau was found to regulate the average radius $<\mathrm{R}^{\mathrm{MT}}>$ of MTs with increasing $\Phi$ (the tau to tubulin-dimer molar ratio in the mixture). Remarkably, $<\mathrm{R}^{\mathrm{MT}}>$ increases rapidly for $0<\phi<0.2$ and saturates for $\phi$ between 0.2 and 0.5 , where $\phi$ is the tau/tubulin-dimer molar ratio for the tau fraction bound to the MT surface (Fig. 6b). The increase in the MT radius results from an increase in the average number of protofilaments in MTs due to a shift in the distribution of MT protofilament numbers $<\mathrm{N}_{\mathrm{pp}}>$ ) (Fig. 6c). The data imply that MAP tau regulates the shape of protofilaments leading to changes in the microtubule diameter.
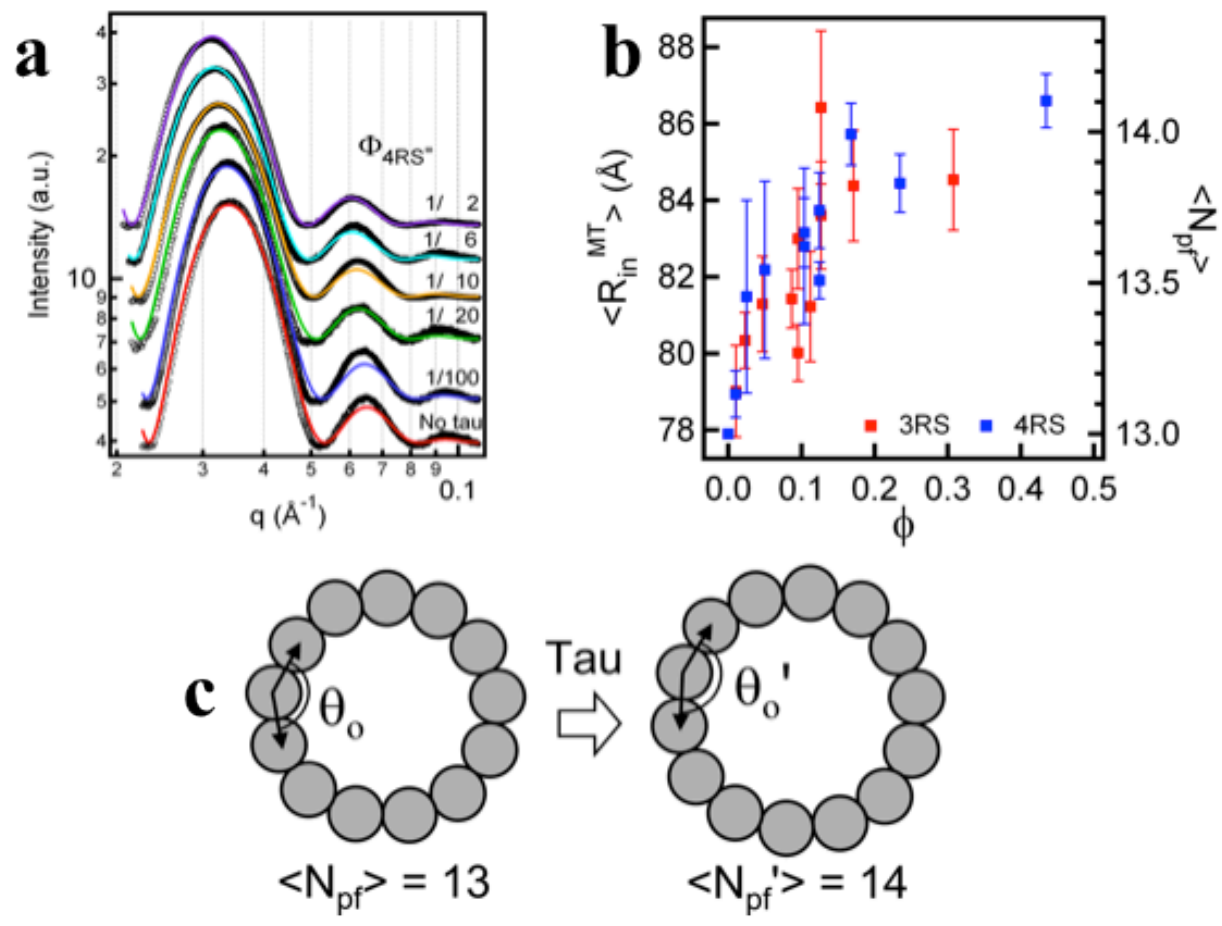

Fig. 6. Microtubule-associated protein (MAP) Tau regulates the diameter of paclitaxel-stabilized microtubules (MTs). a) Synchrotron SAXS results of MTs with bound 4RS Tau as a function of Tau to tubulin-dimer molar ratio in the mixture $(\Phi)$. The SAXS profiles shift to lower q with increasing Tau coverage implying an increase in the MT radius. Colored lines are results of fits of the data to the MT form factor consisting of a hollow tube with the inner radius $\left\langle\mathrm{R}_{\text {in }}{ }^{\mathrm{MT}}>\right.$ the fitting parameter (wall thickness fixed at $4.9 \mathrm{~nm}$ ). b) The inner radius $<\mathrm{R}_{\mathrm{in}}{ }^{\mathrm{MT}}>$ (shown for 4RS-, 3RS- Tau isoforms) increases with increasing $\phi$, the Tau/tubulin-dimer molar ratio for the Tau fraction bound to the MT surface. c) Cross-sections of two MTs showing that with increasing Tau binding the distribution of protofilaments in MTs shifts towards MTs with larger protofilament 
number $\left(\mathrm{N}_{\mathrm{pf}}\right)$ which increases $<\mathrm{R}_{\mathrm{in}}{ }^{\mathrm{MT}}>$. Reprinted from [19] with permission from Cell Press and Biophysical Society.

\section{Potential nanotechnological applications with lipid nanotubes and nanorods with dimensions similar to microtubules}

Aside from the scientific interest in understanding the assembling behavior of MTs in the presence of oppositely charged ions and associated proteins, microtubules are model nanotubes and thus of high interest in applications. Nanotubes are indispensable components in the development of future miniaturized materials. They have applications in diverse areas including as chemical, drug, and gene encapsulation vehicles. However, because of problems related to protein denaturation it is desirable to develop other nanoscale assemblies (rods, tubes) using more robust biomolecules. Here, we briefly review studies by Alexandra Zidovska and collaborators on the formation of a new class of liposomes, termed block liposomes (BLs) [68-70], in mixtures of the highly charged curvature-stabilizing multivalent lipid MVLBG2 (+16e) (Fig. 7a) [71] and zwitterionic lipid DOPC. Hallmarks of BLs are distinctly shaped liposomes, which are linked into a stable structure. BLs include large vesicles attached to rigid cylindrical micelles (Fig. 7b, where arrows point to highly charged and rigid cylindrical micelles). Fig. 7c-e show cartoons of this sphere-rod diblock liposome where the large headgroup MVLBG2 lipid is the minority component and the lipid chains are in the chain melted state. Block liposomes may also consist of micron scale spherical liposomes with stable nanometer scale long tubulular protrusions [68].

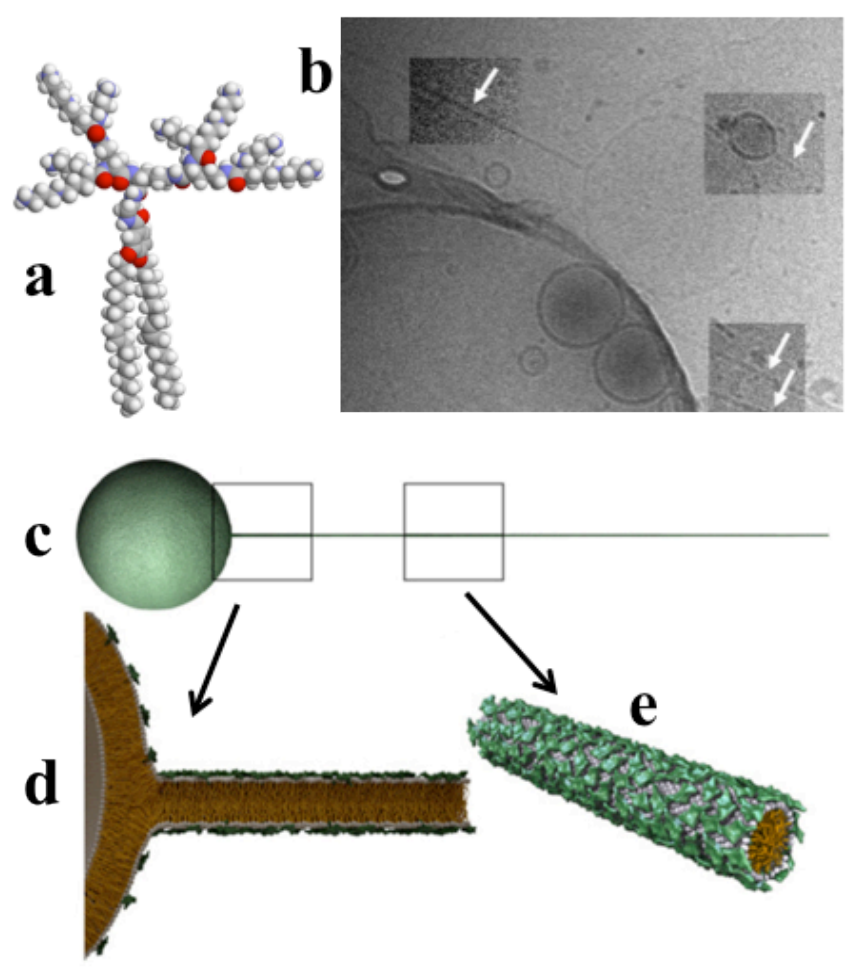

FIG. 7. Example of "sphere-rod" diblock liposome. a) Chemical structure of a dendritic lipid (MVLBG2) bearing 16 positive charges. b) Cryogenic-TEM of diblock (sphere-rod) liposomes. 
White arrows point to nanorods. Lipid nanorods are rigid cylindrical micelles with diameter equal the thickness of a lipid bilayer $(\approx 4 \mathrm{~nm})$ and length up to several microns. c) Cartoon of a MVLBG2/DOPC diblock comprised of a lipid nanorod attached to a vesicle. d) and e) Molecularscale schematics showing the vesicle-micelle junction (d) and a section of the rigid micellar rod (e). The large MVLBG2 headgroups are shown green. Reprinted with permission from [68]. Copyright 2009 American Chemical Society.

Comprehensive characterization studies of BLs by Zidovska et al. led to a model for their formation that suggests an interplay of membrane undulations and concentration fluctuations of the two lipid components [68]. This is expected to lead to phase separation of MVLBG2 and DOPC, with MVLBG2 aggregating into the nanotube or nanorod regions of high curvature, and DOPC in locally flat bilayer spheres attached to the ends of nanotubes/nanorods. In a recent self-consistent field theory Greenall and Gompper have indeed found that mixtures of amphiphiles with different molecular shapes may form equilibrium block liposome phases [72]. One could imagine interesting applications based on BLs. For example, the incorporation of functional biomolecules, would lead to bioactive liquid nanotubes for a range of applications including sensing and chemical delivery. The nanorods may also have potential as templates for needles (e.g. for high resolution AFM imaging) or nanowires.

Before concluding this section we point to another set of interesting recent experiments, where in response to divalent cation binding, distinctly shaped in-plane microphase domains are observed in giant vesciles and cylindrical micelles containing polyvalent amphiphiles $[73,74]$. In these experiments the divalent ligand was observed to induce novel patterns including spotted vesicles, striped micelles and Janus assemblies. It would be interesting to look for similar pattern formation effects in block liposomes (which also contain neutral and multivalent charged amphiphiles) in the presence of oppositely charged multivalent counterions.

\section{Concluding Remarks and Future Directions}

In this review we have described experiments, which show notable ion specific effects in studies designed to elucidate the nature of forces between MTs in the presence of multivalent counter-ions. Divalent cations have two distinct effects on MTs. Those with ionic radii larger than or equal to $0.1 \mathrm{~nm}$ assemble MTs into linear string-like bundles including those with branched structures. On the other hand, smaller ions with radii ranging between $0.072 \mathrm{~nm}$ and $0.083 \mathrm{~nm}$ destabilize MTs most likely by rupturing interprotofilament electrostatic ionic bonds stabilizing the MT wall. Interestingly, the MT destabilizing ion concentration systematically decreases with increasing atomic number for divalent cations. We also reviewed studies, which show that cations with valence equal to or larger than three induce tightly bound MT bundles in sharp contrast to the open structure linear bundles observed with divalent cations. Aside from the charge of the cation in producing bundles with different symmetry, the shape or structure of the multivalent cation (spherical, linear, branched, dendritic, etc) may also play a crucial role in determining bundle symmetry. Further experimental studies are needed to clarify the separate effects of counter-ion valence and chemical structure on ordering. 
We note that experiments involving other biological polyelectrolytes in the presence of counter-ions are known to exhibit attractions and bundle formation in an ion-specific manner. For example, bundling of DNA in solution typically requires counter-ions with valence equal or larger than three. In landmark studies Victor Bloomfield and collaborators have shown that in addition to the valence, the chemical structure of the condensing multivalent counter-ion impacts the overall shape of condensed DNA (torroidal versus rod shaped) [75]. The bundling behavior of DNA electrostatically bound to membranes (i.e. 2D DNA) is qualitatively different from DNA in 3D solution. DNA may be made to spontaneously assemble with cationic membranes in a multilayer structure [76-79]. These nanoparticle condensates are currently employed in gene delivery applications [80-87]. It was found that divalent cations are sufficient to bundle 2D DNA adsorbed on membranes [88]. Remarkably, ion specific effects dominate for such a system where the critical concentration for bundling is observed to decrease with increasing atomic number for $\mathrm{MgCl}_{2}, \mathrm{CaCl}_{2}, \mathrm{MnCl}_{2}$, and $\mathrm{CoCl}_{2}$. Additionally, ion specific bundling has also been reported for the cytoskeletal filamentous actin in the presence of divalent counterions [89-94].

The microtubule-based studies described in this review employed paclitaxel-stabilized MTs, which is a non-biological system. In order to better understand MT ordering in vivo it is important to conduct studies in the absence of paclitaxel. By replacing paclitaxel with the fuel molecule GTP at $37{ }^{\circ} \mathrm{C}$ future studies will help clarify the effects of multivalent ions on MT ordering and disordering in an out of equilibrium "locally" dissipative system (i.e. where tubulin, the building block of MTs, dissipates energy by GTP hydrolysis).

\section{Acknowledgements}

Research supported by the USA Department of Energy (DOE), Office of Science, Basic Energy Sciences (BES), Division of Materials Sciences and Engineering under award number DE-FG02-06ER46314 (polyelectrolyte-directed assembly in filamentous protein systems described in section 4), the USA National Science Foundation (NSF) under award number DMR-1401784 (protein phase behavior), and the USA National Institutes of Health under award number R01-GM 59288 (synthesis of cationic lipid molecules described in section 5). M.C.C. was supported by the National Research Foundation of Korea under awards NRF 2011-0031931 and NRF-2014R1A1A2A16055715. The X-ray diffraction work was carried out at the Stanford Synchrotron Radiation Lightsource, a Directorate of SLAC National Accelerator Laboratory and an Office of Science User Facility operated for the US DOE Office of Science by Stanford University. 


\section{References}

[1] Alberts B et al.: Molecular Biology of the Cell, 4th Ed. Garland Science; 2002.

[2] Mitchison T, Kirschner M. Dynamic instability of microtubule growth. Nature 1984;312:237.

[3] Desai A, Mitchison T. Microtubule polymerization dynamics. Annu Rev Cell Dev Biol 1997;13:83.

[4] Bray D: Cell Movements: From Molecules to Motility, $2^{\text {nd }}$ Ed. Garland; 2001.

[5] Kandel ER, Schwartz JH, Jessell TM: Principles of Neural Science, $4^{\text {th }}$ Ed. McGraw Hill; 2000.

[6] Mandelkow EM, Mandelkow E, Milligan, RA. Microtubule Dynamics and Microtubule Caps: A Time-Resolved Cryo-Electron Microscopy Study. J Cell Bio 1991;114:977.

[7] Schiff PB, Fant J, Horwitz SB. Promotion of tubulin assembly in vitro by taxol. Nature 1979;277:665.

[8] Derry WB, Wilson L, Jordan MA. Substoichiometric binding of Taxol suppresses microtubule dynamics. Biochemistry 1995;34:2203.

[9] Jordan MA, Wilson L. Microtubules as a target for anticancer drugs. Nature Reviews Cancer 2004;4:253.

[10] Nogales E, Wolf SG, Khan IA, Luduena RF, Downing KH. Structure of tubulin at $6.5 \AA$ and location of the taxol-binding site. Nature 1995;375:424.

[11] Elie-Caille C, et al. Straight GDP-tubulin protofilaments form in the presence of taxol. Curr Biol 2007;17:1765.

[12] Ojeda-Lopez MA, Needleman DJ, Song C, Ginsburg A, Kohl P, Li Y, Miller HP, Wilson L, Raviv U, Choi MC, Safinya CR. Transformation of taxol-stabilized microtubules into inverted tubulin tubules triggered by a tubulin conformation switch. Nature Materials 2014;13:195.

[13] Oosawa F, Interaction Between Parallel Rodlike Macroions. Biopolymers 1968;6:1633.

[14] Manning GS. Limiting laws and counterion condensation in polyelectrolyte solutions. I. Colligative properties, J Chem Phys 1969;51:924.

[15] Gelbart WM, Bruinsma RF, Pincus PA, Parsegian VA. DNA-Inspired Electrostatics. Phys Today 2000;53:38.

[16] Grønbech-Jensen N, Mashl RJ, Bruinsma RF, Gelbart WM. Counterion-Induced Attraction Between Rigid Polyelectrolytes. Phys Rev Lett 1997;78:2477. 
[17] Ha BY, Liu AJ. Counterion-mediated Attraction between Two Like-Charged Rods. Phys Rev Lett 1997;79:1289.

[18] Evans DF and Wennerström H: The Colloidal Domain Where Physics, Chemistry, Biology, and Technology Meet, $2^{\text {nd }}$ Ed. Wiley-VCH; 1999.

[19] Choi MC, et al. Human microtubule-associated-protein tau regulates the number of protofilaments in microtubules: a synchrotron x-ray scattering study. Biophys J 2009; 97:519.

[20] Raviv U, Nguyen T, Ghafouri R, Needleman DJ, Li Y, Miller HP, Wilson, Bruinsma RF, Safinya CR. Microtubule protofilament number is modulated in a stepwise fashion by the charge density of an enveloping layer. Biophys J 2007;92:278.

[21] Raviv U, Needleman DJ, Li Y, Miller HP, Wilson L, Safinya CR. Cationic liposomemicrotubule complexes: Pathways to the formation of two-state lipid-protein nanotubes with open or closed ends. Proc Natl Acad Sci USA 2005;102:11167.

[22] Needleman DJ, Ojeda-Lopez MA, Raviv U, Ewert K, Jones JB, Miller HP, Wilson L, Safinya CR. Synchrotron X-ray diffraction study of microtubules buckling and bundling under osmotic stress: A probe of interprotofilament interactions. Phys Rev Lett 2004;93:198104.

[23] Needleman DJ, Ojeda-Lopez MA, Raviv U, Ewert K, Miller HP, Wilson L, Safinya CR. Radial compression of microtubules and the mechanism of action of taxol and associated proteins. Biophys J 2005;89:3410.

[24] Kjellander R, Marcelja S, Quirk JP. Attractive double-layer interaction between calcium clay particles. J Colloid Interface Sci 1988;126:194.

[25] Wennerström H, Khan A, Lindman B. Ionic Surfactants with Divalent Counterions. Adv Coll Int Sci 1991;34:433.

[26] Kékicheff P, Marcelja S, Senden TJ, Shubin VE. Charge reversal seen in electrical double layer interaction of surfaces immersed in 2:1 calcium electrolyte. J Chem Phys 1993;99:6098.

[27] Guldbrand L, Jönsson B, Wennerström H, Linse P. Electrical double layer forces. A Monte Carlo study. J Chem Phys 1984;80:2221.

[28] Bratko D, Jönsson B, Wennerström H. Electrical double layer interactions with image charges. Chem Phys Lett 1986;128:449.

[29] Valleau JP, Ivkov R, Torrie GM. Colloid stability: The forces between charged surfaces in an electrolyte. J Chem Phys 1991;95:520.

[30] Netz RR. Electrostatics of counter-ions at and between planar charged walls: From Poisson-Boltzmann to the strong-coupling theory. Eur Phys J E 2001;5:557.

[31] Netz RR, Orland H. Beyond Poisson-Boltzmann: Fluctuation effects and correlation functions. Eur Phys J E 2000;1:203.

[32] Attard P, Kjellander R, Mitchell DJ. Interactions between Electro-neutral Surfaces bearing mobile charges. Chem Phys Lett 1987;139:219. 
[33] Ha BY, Liu AJ. Counterion-Mediated Attraction between Two Like-Charged Rods. Phys Rev Lett 1997;79:1289.

[34] Pincus P, Safran SA. Charge Fluctuations and Membrane Attractions. Europhys Lett 1998;42:103.

[35] Lukatsky DB, Safran SA. Pressure of Correlated Layer-Charge and Counterion Fluctuations in Charged Thin Films. Phys Rev E 1999;60:5848.

[36] Rouzina I, Bloomfield VA. Macroion Attraction Due to Electrostatic Correlation between Screening Counterions. 1. Mobile Surface-Adsorbed Ions and Diffuse Ion Cloud. J Phys Chem 1996;100:9977.

[37] Shklovskii BI. Wigner Crystal Model of Counterion Induced Bundle Formation of Rodlike Polyelectrolytes. Phys Rev Lett 1999;82:3268.

[38] Arenzon J, Stilck JF, Levin Y. Simple model for attraction between like-charged polyion. Eur Phys J B 1999;12:79.

[39] Lau AWC, Pincus P, Levine D, Fertig HA. Electrostatic attraction of coupled Wigner crystals: Finite temperature effects. Phys Rev E 2001;63:051604.

[40] Baus M, Hansen JP. Statistical mechanics of simple coulomb systems. Phys Rep 1980;59(1):1.

[41] Kanduc M, Naji A, Podgornik R. Counter-ion-mediated weak and strong coupling electrostatic interaction between like-charged cylindrical dielectrics. J Chem Phys 2010;132:224703-1.

[42] Needleman, DJ, et al. Higher-order assembly of microtubules by counterions: From hexagonal bundles to living necklaces. Proc Natl Acad Sci USA 2004;101:16099.

[43] Tuszyński JA, et al. Molecular dynamics simulations of tubulin structure and calculations of electrostatic properties of microtubules. Math Comp Model 2005;41(10):1055.

[44] Needleman, DJ, Ojeda-Lopez MA, Raviv U, Miller HP, Li Y, Song C, Feinstein SC, Wilson L, Choi MC, Safinya CR. Ion specific effects in bundling and depolymerization of taxol-stabilized microtubules. Faraday Discussions 2013;166:31.

[45] Data from Wikipedia on the subject of "ionic radius", 2015

[46] Israelachvili JN: Intermolecular \& Surface Forces. Academic Press; 1992.

[47] Kunz W (editor), Specific Ion Effects. World Scientific Publishing Company; 2010.

[48] Nogales E, Whittaker M, Milligan RA, Downing KH. High-resolution model of the microtubule. Cell 1999;96:79.

[49] Nogales E, Wang HW, Niederstrasser H, Tubulin rings: which way do they curve. Curr Opin Struct Biol 2003;13:256.

[50] Larsson M, Wallin M, Edstrom A. Induction of a sheet polymer of tubulin by Zn 2+. Exp. Cell. Res. 1976;100:104.

[51] Crepeau RH, McEwen B, Dykes G, Edelstein SJ. Structural studies on porcine brain tubulin in extended sheets. J. Mol. Biol. 1977;116:301. 
[52] Tamm LK, Crepeau RH, Edelstein SJ. Three-dimensional reconstruction of tubulin in zinc-induced sheets. 1979;130:473.

[53] Nogales E, Wolf SG, Downing KH. Structure of the $\alpha \beta$ tubulin dimer by electron microscopy. 1998;391:199.

[54] Trinczek B, Biernat J, Baumann K, Mandelkow EM, Mandelkow E. Domains of Tau protein, differential phosphorylation, and dynamic instability of microtubules. Mol Biol Cell 1995;6(12):1887.

[55] Huang Y, Mucke L. Alzheimer's Mechanisms and Therapeutic Strategies. Cell 2012;148(6):1204.

[56] Kosik KS, Joachim CL, Selkoe DJ. Microtubule-associated protein Tau $(\tau)$ is a major antigenic component of paired helical filaments in Alzheimer disease. Proc Natl Acad Sci USA 1986;83(11):4044.

[57] Hutton M, et al. Association of missense and 5'-splice-site mutations in Tau with the inherited dementia FTDP-17. Nature 1998;393(6686):702.

[58] Clark LN, et al. Pathogenic implications of mutations in the Tau gene in pallidoponto-nigral degeneration and related neurodegenerative disorders linked to chromosome 17. Proc Natl Acad Sci USA 1998;95(22):13103.

[59] Spillantini MG et al. Mutation in the Tau gene in familial multiple system Tauopathy with presenile dementia. Proc Natl Acad Sci USA 1998;95(13):7737.

[60] McKee AC, et al. Chronic Traumatic Encephalopathy in Athletes. J Neuropathol Exp Neurol 2009;68(7):709.

[61] Himmler A, Drechsel D, Kirschner MW, Martin DW. Tau consists of a set of proteins with repeated C-terminal microtubule-binding domains and $\mathrm{N}$-terminal domains. Mol Cell Biol 1989;9(4):1381.

[62] Lee G, Cowan N, Kirschner MW. The primary structure and heterogeneity of Tau protein from mouse brain. Science 1988;239(4837): 285.

[63] Butner KA, Kirschner MW. Tau protein binds to microtubules through a flexible array of distributed weak sites. J Cell Biol 1991;115(3):717.

[64] Lee G, Neve RL, Kosik KS. The microtubule binding domain of Tau protein. Neuron 1989;2(6):1615.

[65] Goode BL, Feinstein SC. Identification of a novel microtubule binding and assembly domain in the developmentally regulated inter-repeat region of Tau. J Cell Biol 1994;124(5):769.

[66] Chen J, Kanai Y, Cowan NJ, Hirokawa N. Projection domains of MAP2 and Tau determine spacings between microtubules in dendrites and axons. Nature 1992;360(6405):674.

[67] Kanai Y, Chen, J, Hirokawa N. Microtubule bundling by tau proteins in vivo: analysis of functional domains. EMBO J 1992;11(11):3953. 
[68] Zidovska A, Ewert KK, Quispe J, Carragher B, Potter CS, Safinya CR. Block Liposomes from Curvature-Stabilizing Lipids: Connected Nanotubes, -rods or spheres. Langmuir 2009;25:2979.

[69] Zidovska A, Ewert KK, Quispe J, Carragher B, Potter CS, Safinya CR. The effect of salt and $\mathrm{pH}$ on block liposomes studied by cryogenic transmission electron microscopy. Biochim Biophys Acta - Biomembranes. 2009;1788:1869.

[70] Zidovska A, Ewert KK, Quispe J, Carragher B, Potter CS, Safinya CR. Block Liposome and Nanotube Formation is a General Phenomenon of Membranes Containing Multivalent Lipids. Soft Matter 2011;7:8363.

[71] Ewert KK, Evans HM, Zidovska A, Bouxsein NF, Ahmad A, Safinya CR. A columnar phase of dendritic lipid-based cationic liposome-DNA complexes for gene delivery: Hexagonally ordered cylindrical micelles embedded in a DNA honeycomb lattice. J Am Chem Soc 2006;128:3998.

[72] Greenall MJ, Gompper G. Bilayers Connected by Threadlike Micelles in Amphiphilic Mixtures: A Self-Consistent Field Theory Study. Langmuir 2011;27:3416.

[73] Christian DA, Tian A, Ellenbroek,WG, Levental I, Rajagopal K, Janmey PA, Liu AJ, Baumgart T, Discher DE. Spotted vesicles, striped micelles and Janus assemblies induced by ligand binding. Nature Materials 2009; 8:843.

[74] Levental I, Christian DA, Wang Y-H, Madara JJ, Discher DSE, Janmey PA. Calcium-dependent lateral organization in phosphatidylinositol 4,5-biphosphate (PIP2)- and cholesterol-containing monolayers. Biochemistry 2009;48:8241.

[75] Plum GE, Arscott PG, Bloomfield VA. Condensation of DNA by trivalent cations: Effects of cation structure. Biopolymers 1990;30:631.

[76] Rädler JO, Koltover I, Salditt T, Safinya CR. Structure of DNA-cationic liposome complexes: DNA intercalation in multilamellar membranes in distinct interhelical packing regimes. Science 1997;275:810.

[77] Koltover I, Salditt T, Rädler JO, Safinya CR. An inverted hexagonal phase of cationic liposome-DNA complexes related to DNA release and delivery. Science 1998;281:78.

[78] Koltover I, Salditt T, Safinya CR. Phase diagram, stability, and overcharging of lamellar cationic lipid-DNA self-assembled complexes. Biophys J 1999;77:915.

[79] Safinya CR. Structures of lipid-DNA complexes: supramolecular assembly and gene delivery. Curr Opin Struct Biol 2001;11:440.

[80] Ewert, KK, et al. In Bielke W, Erbacher C (Editors), Topics in Current Chemistry, Vol. 296: Nucleic Acid Transfection. Springer; 2010. Page 191.

[81] Ewert KK, Zidovska A, Ahmad A, Bouxsein NF, Evans HM, McAllister CS, Samuel CE, Safinya CR. Cationic Lipid-Nucleic Acid Complexes for Gene Delivery and Silencing: Pathways and Mechanisms for Plasmid DNA and siRNA. Topics Curr Chem 2010;296:191. 
[82] Huang L, Hung MC, Wagner E (Editors). Advances in Genetics, Vol. 53: Non-Viral Vectors for Gene Therapy, $2^{\text {nd }}$ ed., Part I. Elsevier; 2005.

[83] Lin AJ, Slack NL, Ahmad A, George CX, Samuel CE, Safinya CR. Threedimensional imaging of lipid gene-carriers: Membrane charge density controls universal transfection behavior in lamellar cationic liposome-DNA complexes. Biophys J 2003;84:3307.

[84] Ahmad A, Evans HM, Ewert K, George CX, Samuel CE, Safinya CR. New multivalent cationic lipids reveal bell curve for transfection efficiency versus membrane charge density: lipid-DNA complexes for gene delivery. J Gene Med 2005;7:739.

[85] Bouxsein NF, McAllister CS, Ewert KK, Samuel CE, Safinya CR. Structure and gene silencing activities of monovalent and pentavalent cationic lipid vectors complexed with siRNA. Biochemistry 2007;46:4785.

[86] Safinya CR, Ewert KK. Materials chemistry: Liposomes derived from molecular vases. Nature 2012;489:372.

[87] Safinya CR, Ewert KK, Majzoub RN, Leal C. Cationic liposome-nucleic acid complexes for gene delivery and gene silencing. New J Chem 2014;38:5164.

[88] Koltover I, Wagner K, Safinya CR. DNA condensation in two dimensions. Proc Natl Acad Sci USA 2000;97:14046.

[89] Tang JX, P. A. Janmey. The polyelectrolyte nature of F-actin and the mechanism of actin bundle formation. J Bio Chem 1996;271:8556.

[90] Wong GCL, Lin A, Tang JX, Li Y, Janmey PA, Safinya CR. Lamellar phase of stacked two-dimensional rafts of actin filaments. Phys Rev Lett 2003;91:018103.

[91] Ikawa T, Hoshino F, Watanabe O, Li Y, Pincus P, Safinya CR. Molecular scale imaging of F-actin assemblies immobilized on a photopolymer surface. Phys Rev Lett 2007;98:018101.

[92] Angelini TE, Liang H, Wriggers HW, Wong GCL. Like-charge attraction between polyelectrolytes induced by counterion charge density waves. Proc Natl Acad Sci USA $2003 ; 100: 8634$.

[93] Wong GCL. Electrostatics of rigid polyelectrolytes. Curr Opin Colloid Interface Sci 2006;11:310.

[94] Wong GCL, Pollack L. Electrostatics of strongly charged biological polymers: ionmediated interactions and self-organization in nucleic acids and proteins. Annu Rev Phys Chem 2010;61:171. 